\title{
High Resolution End Group Determination of Low Molecular Weight Polymers by Matrix- Assisted Laser Desorption Ionization on an External Ion Source Fourier Transform Ion Cyclotron Resonance Mass Spectrometer
}

\author{
G. J. van Rooij, M. C. Duursma, R. M. A. Heeren, J. J. Boon, and \\ C. G. de Koster \\ Unit for Macromolecular Mass Spectrometry, FOM-Institute for Atomic and Molecular Physics, Kruislaan 407, \\ 1098 SI Amsterdam, The Netherlands
}

\begin{abstract}
Matrix-assisted laser desorption ionization was performed on an external ion source Fourier transform ion cyclotron resonance mass spectrometer equipped with a 7-T superconducting magnet to analyze end groups of synthetic polymers in the mass range from 500 to $5000 \mathrm{u}$. Native, perdeutero methylated, propylated, and acetylated polyethylene glycol and polyvinyl pyrrolidone with unknown end-group elemental composition were investigated in the mass range up to $5000 \mathrm{u}$ by using a 2,5-dihydroxybenzoic acid matrix. A small electrospray setup was used for the deposition of the samples. Two methods to process data were evaluated for the determination of end groups from the measured masses of the component molecules in the molecular weight ranges: a regression method and an averaging method. The averaging method is demonstrated to allow end-group mass determinations with an accuracy within $3 \mathrm{mu}$ for the molecular weight range from 500 to 1400 and within $20 \mathrm{mu}$ for the molecular weight range from 3400 to 5000 . This is sufficient to identify the elemental composition of end groups in unknown polymer samples. (J Am Soc Mass Spectrom 1996, 7, 449-457)
\end{abstract}

$\mathrm{I}$ nsight into the functional properties of polymers and a verification of the reaction products of polymerization processes can be obtained from knowledge of the structure of the polymer end groups. In this study we present the possibility to determine end groups of polymers by matrix-assisted laser desorption and ionization (MALDI) on an external ion source Fourier transform ion cyclotron resonance mass spectrometer (ext-FTICR-MS).

Most of the commonly used techniques to characterize synthetic polymers, such as osmometry, cryoscopy, end-group titration, and light scattering [1], are capable only of yielding an average molecular weight. Other methods, such as size-exclusion chromatography and high-performance liquid chromatography, are able to separate the oligomeric components of the polymer system, but with limited resolution and accuracy [2]. Consequently, these techniques are not suitable for the determination of exact molecular weight distributions of the individual components of the polymer molecular weight distribution. In recent years it has been shown that mass spectrometric analysis can provide

Address reprint requests to Dr. J. J. Boon, Kruislaan 407. 1098 SJ Amsterdam, The Netherlands. this information [3-10]. For mass spectrometric characterization of polymer systems, soft ionization techniques are commonly used to lift and convert the large molecules from the condensed phase to gas phase (quasi) molecular ions. The advantage of these techniques is that they minimize fragmentation of the molecular ions, even for high molecular weights, which is imperative for the measurement of the molecular weight distribution. Various "soft" ionization techniques have heen used: secondary ion mass spectrometry [3], plasma desorption [4], fast-atom bombardment [5], laser desorption [6], electrospray ionization [7], and MALDI. Of these techniques, especially MALDI has evolved as an important ionization technique for molecular weight determinations of synthetic and biopolymers [8-10]. However, the low resolution of the widely used MALDI time-of-flight (TOF) mass spectrometers limits the mass measurement accuracy for higher molecular weight samples. Because in general the mass of a polymer is much larger than the mass of its end group, ultra high mass accuracy is required for an end-group determination. TOF mass spectrometers are inadequate for such determinations. This problem can be overcome by coupling MALDI to a Fourier transform ion cyclotron resonance mass 
spectrometer (FTICR-MS) - an established method to achieve ultra high mass resolution and perform accurate mass measurements [11-17].

In most MALDI-FTICR-MS studies the MALDI process takes place near the ion ICR cell. In such configurations difficulties are encountered in trapping the MALDI ions inside the ICR cell because of the large kinetic energy of the laser-desorbed ions. Several experimental and instrumental approaches have been tried to improve the trapping efficiency, such as a gated trapping deceleration protocol [18] and collisional cooling [19, 20]. Mclver et al. [21-23] described an external ionization method where the MALDI source was outside the magnetic field. MALDI ions were transferred by an rf-only quadrupole ion guide and injected into the center of the ICR cell. Their experiments have shown that the external ion source configuration provides improved mass measurement accuracy and mass resolution for MALDI-produced ions $[24,25]$.

The hardware for the experiments in our study is an in-house modified commercially available external ion source 7-T FTICR-MS instrument. First results on endgroup determinations that used this instrument have been reported by de Koster et al. [26]. In this article measurements on native, perdeutero methylated, propylated, and acetylated polyethylene glycol (that have known end-group structures) as well as measurements on polyvinyl pyrrolidone (with unknown endgroup elemental composition) are presented. We have used these measurements to compare two different methods for end-group mass determination from exact mass measurements of the components of a polymer molecular weight distribution. Error analysis is carried out to test the accuracy of the methodology.

\section{Experimental}

\section{Instrumental}

The MALDI experiments were performed on the FOM APEX-HR1, an external ion source FTICR-MS that is a modified Bruker Spectrospin (Fällanden, Switzerland) APEX 7.0e FTICR-MS [27]. Samples for the MALDI experiments were deposited on the stainless steel tip of a direct insertion probe. This probe was inserted into the external ion source via a vacuum lock. The ion source was pumped to typically $1 \times 10^{-7}$ mbar by using a combination of a turbomolecular and a turbodrag pump backed by an oil-free membrane pump. A total of three pumping stages along with two differential pumping restrictions maintain an ultra high vacuum (UHV) base pressure of $<1 \times 10^{-10}$ mbar in the ICR-cell region. I'he ions produced in the source were transferred by the standard Bruker electrostatic ion optics to the ICR cell. The ions were extracted from the ionization region by using a potential difference of approximately $10 \mathrm{~V}$ between the source housing and the first extraction plate. Subsequently, the ions were accelerated to $3 \mathrm{keV}$ to prevent radial ejection in the inhomogeneous stray field of the magnet. After deceleration to approximately $1 \mathrm{eV}$ the ions were trapped in the electrostatic potential well between two trap electrodes of an Infinity ${ }^{\mathrm{IM}}$ Cell (Bruker-Spectrospin, Fällanden, Switzerland) [28], which is centered inside the homogeneous region of the 7-T superconducting magnet. Control of the measurements on the FTICR-MS and data acquisition and processing were performed by the Bruker software XMASS running on an SGI Indigo R4000 (Silicon Graphics, Mountain View, CA) UNIX-based workstation.

A Photon Technology PL2300 (London, Ontario, Canada) nitrogen laser was used to produce 337.1-nm laser pulses with an energy of $1.3 \mathrm{~mJ}$ and a pulse length of $600 \mathrm{ps}$. The laser beam was transferred to the source over a distance of $2 \mathrm{~m}$ and focused onto the MALDI probe with an incident angle of $45^{\circ}$ by using an optical system that consisted of two telescopes (two pairs of biconvex lenses with 300-, 400-, 40-, and 80-mm focal length, respectively). The area of the laser beam at the MALDI probe was $4.5 \mathrm{~mm}^{2}$ determined from the discoloration of photosensitive paper. The transmitted energy per pulse measured inside the ion source $(0.48 \mathrm{~mJ})$ resulted in a fluence of $10.6 \mathrm{~mJ} / \mathrm{cm}^{2}$ (which corresponds to a power density of $17.5 \mathrm{MW} / \mathrm{cm}^{2}$ ) on the MALDI target.

Each experiment started with a quench pulse that removes all ions from the ICR cell. This was followed by a transistor-transistor logic (TTL) pulse that triggered the laser. At the same time the front trapping electrode was set to ground potential to allow the ions to enter the cell. The rear trapping electrode remained at typically $3.5 \mathrm{~V}$. Following a time delay determined by the time-of-flight of the ions in the mass range of interest, the front trapping electrode was set to $2.5 \mathrm{~V}$. The asymmetry in the trapping field was introduced because it gives optimal signals in our experiments. Following a 50-ms delay, the trapped ions were coherently excited to a radius a little under the cell radius by a frequency sweep (chirp) excitation from a frequency synthesizer. To acquire a mass spectrum the ion-image-current signals on the detection plates were digitized by a 12-bit, $20-\mathrm{MHz}$ analog-to-digital converter and stored in a 128 -Kbyte fast memory. Finally, discrete Fourier transformation of this time domain signal followed by magnitude calculation yielded a frequency spectrum, which was transformed to a mass spectrum. All spectra shown are zero-filled to 256 Kbytes.

To calculate the actual mass of an ion with a given elemental composition, the atomic masses tabulated in the 1983 atomic mass table composed by Wapstra et al. [29] were used.

\section{Sample Preparation by Electrospraying}

The compounds examined were polyethylene glycol (PEG) 1000 from Serva (Heidelberg, Germany), 
PEG4000 from Fluka Chemical (Buchs, Switzerland), and polyvinyl pyrrolidone (PVP3000) with unknown end-group structure. For all the measurements 2,5dihydroxybenzoic acid (DHB) from Sigma Chemical Co. (St. Louis, MO) was used as the MALDI matrix.

The native PEGs and the PVP were used without further purification. For the alkylation $80-\mathrm{mg} \mathrm{KOH}$ powder was added to 25-mg PEG and the samples were dried in vacuo over phosphorus pentoxide at $50{ }^{\circ} \mathrm{C}$ for $1 \mathrm{~h}$. The samples were dissolved in 1-mL dry dimethyl sulfoxide and $0.2-\mathrm{mL}$ (methyl- $d_{3}$ ) iodide or propyl iodide was added. The methylation was completed at room temperature in $2 \mathrm{~h}$; the propylation was completed at $60^{\circ} \mathrm{C}$ in $5 \mathrm{~h}$. The reaction was stopped by adding $5 \mathrm{~mL}$ of ice water and the derivatized PEGs were extracted with trichloromethane $(3 \times 2 \mathrm{~mL})$. The combined extracts were washed with water $(3 \times 2$ $\mathrm{mL}$ ). The $\mathrm{CHCl}_{3}$ was evaporated under a stream of nitrogen and the residues were dried in vacuo over phosphorus pentoxide at $50{ }^{\circ} \mathrm{C}$ for $1 \mathrm{~h}$. 'I The acetylated sample was prepared by dissolving 30-mg PEG in 1 -mL dry pyridine. Acetic anhydride was added and the sample was allowed to react for $16 \mathrm{~h}$ at $100{ }^{\circ} \mathrm{C}$. The pyridine and acetic anhydride were evaporated under a stream of nitrogen.

The samples for MALDI mass spectrometry were prepared by mixing a 1-M matrix solution in ethanol with approximately $10 \mathrm{-g} / \mathrm{I}$. analyte solution in ethanol. For PEG4000 the molar ratio 4000:1 was found to be optimum, whereas for the other compounds the molar ratio 1000:1 gave the best results. The resulting sample matrix solution was deposited onto a stainless steel probe tip by using a small electrospray setup. In this setup a Harvard Apparatus (South Natick, MA) syringe pump (model 55-1111) is used to deliver 0.30 $\mathrm{mL} / \mathrm{h}$ to a $180-\mu \mathrm{m}$ internal diameter capillary for $20 \mathrm{~min}$. This capillary is electrically isolated from the pump with a PEEK insulator and is typically set to $4 \mathrm{kV}$. The MALDI probe tip is localed about $7 \mathrm{~mm}$ behind the capillary and is set to ground potential. The resulting analyte amount on the probe is approximately $10 \mathrm{ng}$. In comparison with the dried droplet method [8], this method of sample deposition provides smaller crystals, which results in an improvement of signal-to-noise ratio by a factor of 2 in our instrument.

\section{Results and Discussion}

\section{Numerical Considerations of End-Group Analysis}

Because of the statistical nature of the polymerization process, most polymeric materials consist of mixlures of molecules of various sizes. Because the MALDI ionization technique leads to protonated or cationized molecules, the measured masses of the component (homo-) polymers in this molecular weight distribution can be written as

$$
M_{\text {meas }}=n \times M_{\text {mon }}+M_{\text {end }}+M_{\text {cat }}
$$

Here $n$ is the degree of polymerization and $M_{\text {mon }}$ $M_{\text {end }}$, and $M_{\text {cat }}$ are the mass of the repeating unit, the residue mass of the end group, and the mass of the cation, respectively. Note that this expression holds only for the ${ }^{12} \mathrm{C}$ monoisotopic peaks in the measured molecular weight distribution. If component polymers that conlain one or more ${ }^{13} \mathrm{C}$ atoms are considered, an additional term has to be introduced that incorporates the mass difference between ${ }^{12} \mathrm{C}$ and ${ }^{13} \mathrm{C}$. Linear regression analysis on the measured molecular weights of an isotopically resolved selected series of cationized component polymer molecules as a function of the degree of polymerization will yield the mass of the repeating unit and the sum of the residue mass of the end group plus the mass of the cation. The slope of the fitted line gives the mass of the repeating unit and the intercept with the $y$ axis will represent the combined mass of the end group and the cation. However, there are two problems inherent to this method: first, the determination of the cation adduct; second, the assignment of the degree of polymerization to the different molecular peaks in the spectra. For many polymer samples the first problem can be solved easily by comparison of a series of measurements where different alkali salts are added to the matrix as dopants. This addition of alkali salts will influence the abundance of the cationized species and it becomes possible to identify the different adducts. The assignment of the degree of polymerization is more ambiguous. A criterion for the assignment of $n$ is that the estimated end-group mass corresponds with elemental compositions that are plausible in the context of reaction mechanisms in polymer synthesis and synthetic routes that lead to the polymer. Consequently one has to evaluate in principle all possible end-group masses given by

$$
M_{\text {end }}=M_{0}+i^{*} M_{\text {mon }}
$$

(in which $M_{0}$ is the smallest possible end-group mass and $i=0,1,2, \ldots$ ).

In the foregoing method both the monomer mass and the end-group mass are determined from measured data. However, it is obvious that an error in the monomer mass determination will introduce a progressive error in the end-group mass because of the extrapolation of the regression line necessary to find the intercept with the $y$ axis. Therefore a significant improvement of the accuracy of the intercept can be obtained when the elemental composition of the repeating unit has been determined. In that case the slope of the line can be fixed to the actual residual monomer mass and only the intercept will be fitted. This method is equivalent to determination of the end group for each individual peak in the spectrum (by subtracting $n$ times the monomer mass from the measured mass) and subsequently averaging the results.

The accuracy in determination of end-group masses according to both methods can be compared by performing an error analysis. The uncertainty in the deter- 
mination of the end-group mass as a result of the (expectation value of the) accuracy of the mass measurements $\sigma_{\text {data }}$ by using the first method-the regression method-is determined by the uncertainty in the estimation of the coefficients $a$ and $b$ in the fit of the straight line $Y=a n+b$ through the set of data points. In this fit $a$ and $b$ reflect the monomer and the endgroup mass, respectively. The errors are found to be [30]

$$
\begin{aligned}
\sigma_{a}^{2} & =\frac{N \sigma_{\text {data }}^{2}}{N \sum_{i=1}^{N} n_{l}^{2}-\left(\sum_{i=1}^{N} n_{i}\right)^{2}}, \\
\sigma_{b}^{2} & =\frac{\sigma_{\text {data }}^{2} \sum_{i=1}^{N} n_{i}^{2}}{N \sum_{i=1}^{N} n_{i}^{2}-\left(\sum_{i=1}^{N} n_{i}\right)^{2}},
\end{aligned}
$$

where $n_{i}$ is the degree of polymerization that corresponds to a measured mass $M_{i}$ and $N$ is the number of data points. The uncertainty in the determination of the end-group mass is equal to $\sigma_{b}$. To compare the uncertainties for the two methods we can simplify eqs 3 by considering the case in which the data points are distributed equally about the origin. This can be obtained for any data set by redefinition of the origin that performs the translation $n^{\prime}=n-\bar{n}$ (where $\bar{n}$ is the average degree of polymerization), which is a linear transformation and therefore does not affect the magnitudes of the uncertainties. After transformation of the data, we find from eqs 3 that in the new coordinate system, the uncertainties in fitting the line $Y^{\prime}=a^{\prime} n^{\prime}+b^{\prime}$ (note that primed symbols refer to the new coordinate system) are given by

$$
\sigma_{a}^{2}=\frac{\sigma_{\text {data }}^{2}}{\sum_{i=1}^{N} n_{i}^{\prime 2}}, \quad \sigma_{b}^{2}=\frac{\sigma_{\text {data }}^{2}}{N} .
$$

Insertion of $n^{\prime}=n-\bar{n}$ into the expression for $\sigma_{a}^{\prime}$ shows that the uncertainty in the parameter $a$ is invariant for linear transformations, that is, $\sigma_{a}^{\prime} \equiv \sigma_{a}$. To find the mass of the end group in the new coordinate system, $n^{\prime}=-\bar{n}$ is substituted into $Y^{\prime}=a^{\prime} n^{\prime}+b^{\prime}$, which results in

$$
M_{\text {end }}=b^{\prime}-a^{\prime} \bar{n} .
$$

The corresponding uncertainty is obtained by calculation of the propagation of errors in the extrapolation [30]

$$
\sigma_{\text {end }}^{2}=\sigma_{b}^{\prime 2}+\bar{n}^{2} \sigma_{a}^{\prime 2}=\frac{\sigma_{\text {data }}^{2}}{N}+\bar{n}^{2} \frac{\sigma_{\text {data }}^{2}}{\sum_{i=1}^{N} n_{i}^{\prime 2}} .
$$

For the second method-the averaging method-the uncertainty in the determination of the end-group mass as calculated from the propagation of errors [30] is given by

$$
\sigma_{\text {end }}^{2}=\frac{\sigma_{\text {data }}^{2}}{N} .
$$

Comparison of eqs 6 and 7 shows that the difference between the accuracies of the two methods is the (progressive) contribution of the uncertainty in the determined monomer mass $\sigma_{a}$. Consequently with a known monomer elemental composition, the averaging method yields higher end-group mass accuracies.

\section{Tests of the Methodology on PEG1000 and PEG4000}

The polymer samples investigated in a first series of experiments were polyethylene glycols with different molecular weight averages. Because these PEGs are reference samples with known polymer structure, system performance could be optimized and characterized in the course of these experiments. Figure 1 shows the MALDI-FTICR-MS spectrum of PEG1000 in broad band (mass range $m / z 500-5000$ ) and two expansions of the mass scale. Sodium iodide was added to the matrix to increase the signal-to-noise $(\mathrm{S} / \mathrm{N})$ ratio. The broadband mass spectrum covers the entire molecular
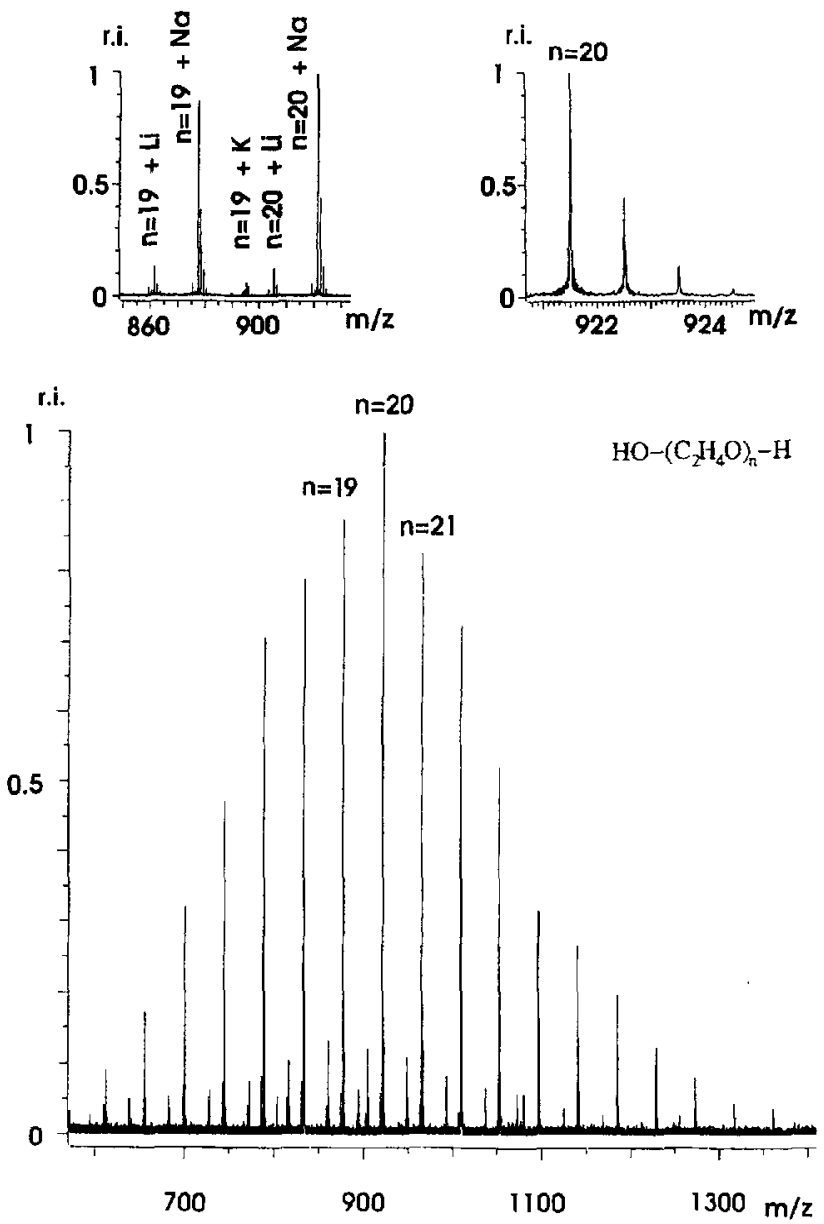

Figure 1. Broadband MALDI-FTICR-MS spectrum of native PEG1000 with sodium iodide added to the matrix. Two expansions of the mass scale are given. The left-hand inset shows the different cation adducts and the right-hand inset shows the high resolution of the measurement $(30,000)$. 
weight distribution of PEG1000. The resolution in broadband mode $(\mathrm{m} / \mathrm{dm})_{50 \%}$ is 30,000 with a $\mathrm{S} / \mathrm{N}$ ratio of 100 at $\mathrm{m} / \mathrm{z} 921$ for a sample amount of $10 \mathrm{ng}$ on target. The right-hand inset shows that the resolution is sufficient to resolve the naturally occurring ${ }^{12} \mathrm{C} /{ }^{13} \mathrm{C}$ isotopes of the component molecules. Therefore no further optimization of the mass resolving power was attempted. The molecular weight distribution is near Gaussian shaped as can be expected from the statistical nature of the polymerization process [31]. The spectrum predominantly shows sodium cationized PEG molecules at equidistant intervals that are nominally $44 \mathrm{u}$. Also lithium and potassium cationized components are present at lower abundances. Matrix adduct ions are not observed. For example, the PEG nonadecamer quasimolecular ions $\left[\mathrm{M}+\mathrm{Li}^{+},[\mathrm{M}\right.$ $+\mathrm{Na}]^{+}$, and $[\mathrm{M}+\mathrm{K}]^{+}$are observed at $m / z$ 861, 877, and 893, respectively (left-hand inset in Figure 1). The alkaline salt contamination from the sample preparation process was sufficient to produce the lithium and potassium cation adducts. To prove the identity of the ionizing species, a series of measurements was performed in which the alkali salts lithium iodide, sodium iodide, and potassium iodide subsequently were added to the matrix (Figure 2). Finally, we have measured the lithium, sodium, and potassium cationized perdeutero methylated PEG1000 molecular weight distributions (spectra not shown). The masses of these peaks are listed in Table 1. The individual components of the molecular weight distributions are measured with a mass accuracy better than $8 \mathrm{ppm}$. We have taken $8 \mathrm{mu}$, which corresponds to this mass accuracy, as the accuracy in the experimental data $\left(\sigma_{\text {data }}\right)$ for the calculation of the uncertainties in the error analysis. This value

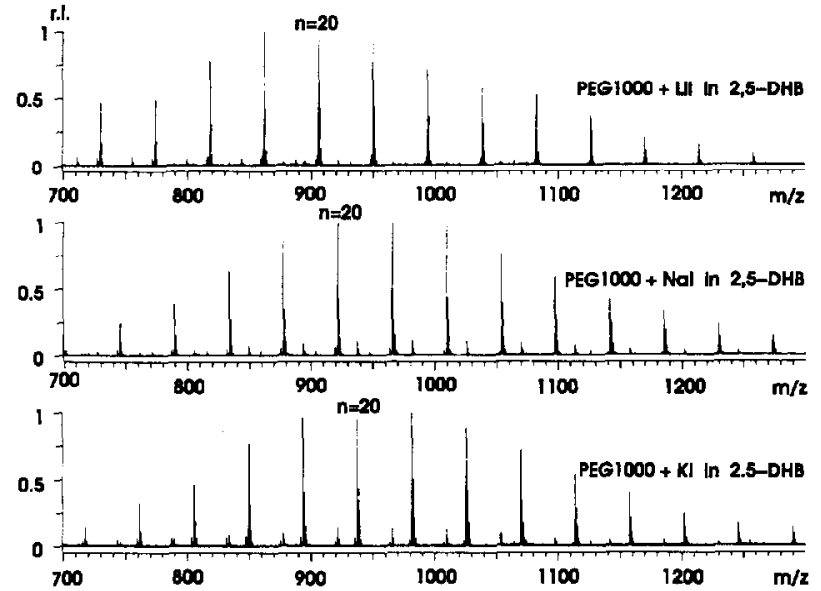

Figure 2. Broadband MALDI-FTICR-MS spectra of PEG1000 with lithium, sodium, and potassium iodide, respectively, added to the matrix.

only changes marginally over the relatively narrow mass range of interest. The measurements on PEG1000 as described in the preceding text were used to test the accuracy of the two methods of end-group determination (eqs 6 and 7). Only the peaks with S/N > 10 were part of the calculations. The results of the analysis described in the previous section are given in Table 2. It is obvious that both methods have an accuracy that is sufficient to confirm the identity of the distal parts of the polymer. As argued in the previous section, the second method yields a higher accuracy for the endgroup mass. The errors in the determined end-group masses are in agreement with the predicted values, given the 8-ppm mass measurement accuracy.

Table 1. The measured masses of the components of the PEG1000 molecular weight distributions and the corresponding degree of polymerization $n[-(\cdots)$ - denotes the chain of PEG monomer units].

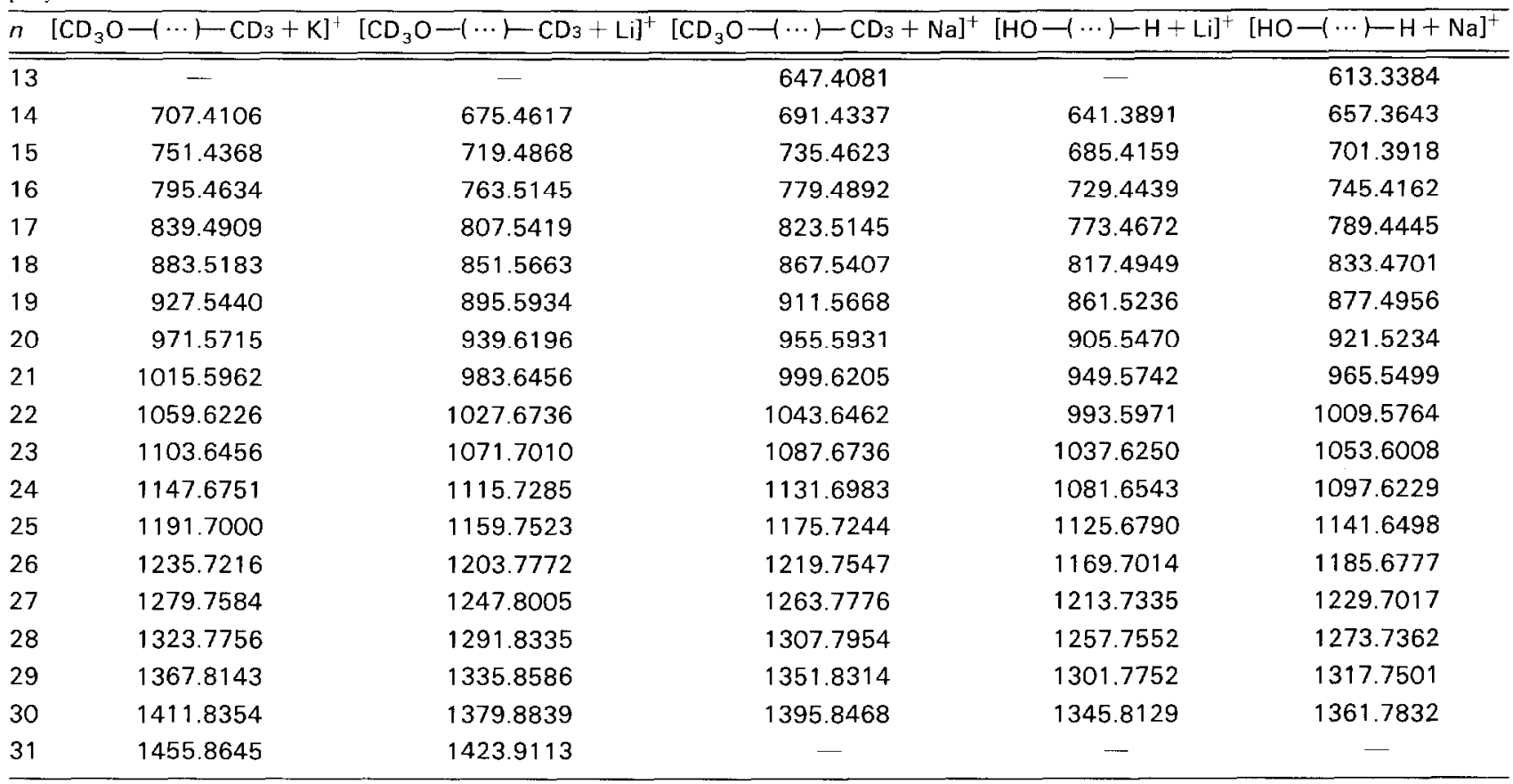


Table 2. Masses and deviations in the analysis of native and perdeutero methylated PEG1000 end groups by MALDI-FTICR-MS ${ }^{a}$

\begin{tabular}{|c|c|c|c|c|c|}
\hline $\begin{array}{l}\text { Masses and } \\
\text { Deviations }(\mathrm{u})\end{array}$ & $\begin{array}{c}{\left[\mathrm{CD}_{3} \mathrm{O}-(\cdots)-\right.} \\
\left.\mathrm{CD}_{3}+\mathrm{K}\right]^{+} \\
\end{array}$ & $\begin{array}{c}{\left[\mathrm{CD}_{3} \mathrm{O}-(\cdots)-\right.} \\
\mathrm{CD}_{3}+\mathrm{Li}^{+} \\
\end{array}$ & $\begin{array}{c}{\left[\mathrm{CD}_{3} \mathrm{O}-(\cdots)-\right.} \\
\left.\mathrm{CD}_{3}+\mathrm{Na}\right]^{+}\end{array}$ & {$\left[\mathrm{HO}-(\cdots)-\mathrm{H}+\mathrm{Li}^{-}\right.$} & {$\left[\mathrm{HO}-(\cdots)-\mathrm{H}+\mathrm{Na}^{+}\right.$} \\
\hline $\bar{M}$ man, artual $_{1}$ & 44.0262 & 44.0262 & 44.0262 & 44.0262 & 44.0262 \\
\hline$M_{\text {man, regression }}$ & 44.0265 & 44.0264 & 44.0261 & 44.0261 & 44.0260 \\
\hline$\Delta M_{\text {mon. regressian }}$ & 0.0003 & 0.0002 & -0.0001 & -0.0001 & -0.0002 \\
\hline$\sigma_{\text {mon. regression }}$ & 0.0004 & 0.0004 & 0.0004 & 0.0004 & 0.0004 \\
\hline$M_{\text {end, actual }}$ & 91.0432 & 59.0955 & 75.0693 & 25.0260 & 40.9998 \\
\hline$M_{\text {end,regression }}$ & 91.0392 & 59.0913 & 75.0708 & 25.0244 & 41.0012 \\
\hline$\Delta M_{\text {end, regression }}$ & -0.0041 & -0.0042 & 0.0015 & -0.0016 & 0.0014 \\
\hline 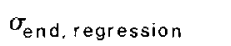 & 0.0084 & 0.0084 & 0.0080 & 0.0089 & 0.0080 \\
\hline$M_{\text {end, average }}$ & 91.0460 & 59.0963 & 75.0685 & 25.0227 & 40.9971 \\
\hline$\Delta M_{\text {end. average }}$ & 0.0028 & 0.0008 & -0.0008 & -0.0034 & -0.0027 \\
\hline$\sigma_{\text {end, average }}$ & 0.0019 & 0.0019 & 0.0019 & 0.0019 & 0.0019 \\
\hline
\end{tabular}

a These results were obtained by using the masses listed in Table $1 . \Delta$ denotes the difference between the actual end-group mass and the estimated mass. The standard deviations $\sigma_{\text {mon }}$ and $\sigma_{\text {end }}$ were calculated from eqs 6 and $7 . \sigma_{\text {data }}$ is taken to be 8 mu. $-\{\ldots\}-$ denotes the chain of PEG monomer units.

After the encouraging results from the PEG1000, experiments were performed on PEG4000. The spectrum of the native PEG4000 is shown in Higure 3. The components of the molecular weight distribution are measured with a mass accuracy better than $15 \mathrm{ppm}$ and their masses are listed in Table 3. The polymer $(n=95)$ at $m / z 4239[\mathrm{M}+\mathrm{K}]^{+}$is resolved with a resolution of 6000 and a $\mathrm{S} / \mathrm{N}$ ratio of 75 . The polymer molecular weight distribution covers the mass range $m / z 3400-5200(\mathrm{~S} / \mathrm{N}>5$ ). To test the accuracy of the methods and the experimental conditions, the dipropylether and the diacetate of PEG4000 were studied in addition to the native PEG4000. The residue masses of

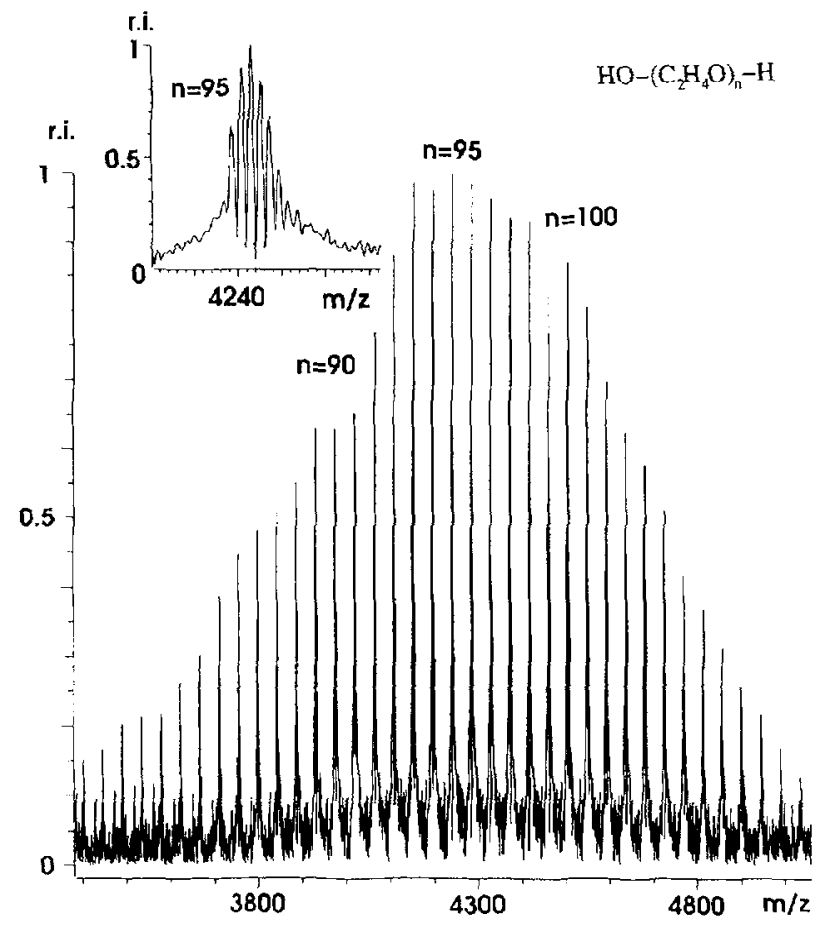

Figure 3. Broadband MALDI-FTICR-MS spectrum of PEG4000. The inset shows the unit mass resolved peak at $m / z 4239$. the dipropyl and diacetate end groups differ only by $72 \mathrm{mu}$. Consequently a resolving power of at least 56,000 at $\mathrm{m} / z 4000$ is required to distinguish the two derivatives. The measured masses of the components of the molecular weight distributions of the derivatized PEG4000 compounds were similar to those from native PEG4000 and also are listed in Table 3. It is obvious that the resolution in broadband mode is not sufficient to resolve the two derivatives in one spectrum. On the other hand, the mass accuracy should be sufficient to distinguish the two derivatives on the basis of end-group mass determinations from different measurements. The results of the analysis of the data are listed in Table 4. The uncertainties in the error analysis are calculated by taking $60 \mathrm{mu}$ as the accuracy in the experimental data $\sigma_{\text {data }}$ (which corresponds to 15 -ppm mass accuracy at $m / z$ 4000). Note that for the end-group analysis the peaks in the spectrum that correspond to polymers that contain two ${ }^{13} \mathrm{C}$ isotopes are used because they have the highest abundance in the mass range around $m / z 4000$. The identification of these peaks is based on caiculated isotopic patterns. The implication of this choice is that the determined end-group masses will be the summation of the endgroup mass, the mass of the cation, and the mass difference between ${ }^{12} \mathrm{C}_{2}$ and ${ }^{13} \mathrm{C}_{2}$. We concluded from the results that the regression method becomes less accurate for higher masses because of the large effect of the extrapolation. Furthermore, the results show that the predicted errors are much higher than the actual errors. This difference is caused by a relatively large variation of $\sigma_{\text {data }}$ over the molecular weight distribution (roughly a factor of 2), that is, the assumption of a constant measurement uncertainty no longer holds. The error from the averaging method still allows the confirmation of the identity of the end groups with an accuracy within $20 \mathrm{mu}$, which means that it is possible to distinguish between the two derivatives with isobaric nominal end-group mass in end-group determinations according to the averaging method. 
Table 3. The measured masses of the components of the PEG4000 molecular weight distributions and the corresponding degree of polymerization $n .[-(\cdots)$ - denotes the chain of PEG monomer units].

\begin{tabular}{|c|c|c|c|}
\hline$n$ & {$\left[\mathrm{HO}-(\cdots)-\mathrm{H}+\mathrm{Na}^{+}\right.$} & $\begin{array}{c}{\left[\mathrm{CH}_{3}(\mathrm{CO})^{\prime}-(\cdots)-\right.} \\
\left.(\mathrm{CO}) \mathrm{CH}_{3}+\mathrm{Na}\right]^{+}\end{array}$ & $\begin{array}{c}{\left[\mathrm{C}_{3} \mathrm{H}_{7} \mathrm{O}-(\cdots)-\right.} \\
\left.\mathrm{C}_{3} \mathrm{H}_{7}+\mathrm{Na}\right]^{+}\end{array}$ \\
\hline 78 & - & 3561.130 & - \\
\hline 79 & - & 3605.151 & - \\
\hline 80 & - & 3649.173 & - \\
\hline 81 & - & 3693.246 & 3693.145 \\
\hline 82 & - & 3737.280 & 3737.182 \\
\hline 83 & - & 3781.298 & 3781.223 \\
\hline 84 & - & 3825.290 & 3825.260 \\
\hline 85 & 3785.265 & 3869.350 & 3869.261 \\
\hline 86 & 3829.291 & 3913.363 & 3913.284 \\
\hline 87 & 3873.255 & 3957.389 & 3957.319 \\
\hline 88 & 3917.258 & 4001.410 & 4001.342 \\
\hline 89 & 3961.342 & 4045.440 & 4045.369 \\
\hline 90 & 4005.355 & 4089.462 & 4089.403 \\
\hline 91 & 4049.355 & 4133.486 & 4133.419 \\
\hline 92 & 4093.393 & 4177.523 & 4177.442 \\
\hline 93 & 4137.418 & 4221.563 & 4221.486 \\
\hline 94 & 4181.472 & 4265.576 & 4265.510 \\
\hline 95 & 4225.486 & 4309.593 & 4309.541 \\
\hline 96 & 4269.525 & 4353.622 & 4353.556 \\
\hline 97 & 4313.557 & 4397.655 & 4397.576 \\
\hline 98 & 4357.574 & 4441.665 & 4441.614 \\
\hline 99 & 4401.540 & 4485.732 & 4485.627 \\
\hline 100 & 4445.613 & 4529.722 & 4529.665 \\
\hline 101 & 4489.715 & 4573.738 & 4573.688 \\
\hline 102 & 4533.703 & 4617.754 & - \\
\hline 103 & - & 4661.813 & - \\
\hline 104 & - & 4705.852 & - \\
\hline 105 & - & 4749.851 & - \\
\hline
\end{tabular}

Table 4. Masses and deviations in the analysis of native, dipropyl, and diacetyl PEG4000 end groups by MALDI-FT'ICR-MS"

\begin{tabular}{|c|c|c|c|}
\hline $\begin{array}{l}\text { Masses and } \\
\text { Deviations } \\
\text { (u) }\end{array}$ & $[\mathrm{HO}-\cdots)-\mathrm{H}+\mathrm{Na}]^{+}$ & $\begin{array}{c}{\left[\mathrm{CH}_{3}(\mathrm{CO})^{-}-(\cdots)\right.} \\
\left(\mathrm{CO}^{-} \mathrm{CH}_{3}+\mathrm{Na}\right]^{+}\end{array}$ & $\begin{array}{c}{\left[\mathrm{C}_{3} \mathrm{H}_{7} \mathrm{O}-(\cdots)\right.} \\
\left.\mathrm{C}_{3} \mathrm{H}_{7}+\mathrm{Na}\right]^{+}\end{array}$ \\
\hline$\overline{M_{\text {mon, actual }}}$ & 44.0262 & 44.0262 & 44.0262 \\
\hline$M_{\text {mon, regression }}$ & 44.0285 & 44.0264 & 44.0264 \\
\hline$\Delta M_{\text {mon, regression }}$ & 0.0023 & 0.0002 & 0.0002 \\
\hline$\sigma_{\text {mon, regression }}$ & 0.0025 & 0.0014 & 0.0020 \\
\hline$M_{\text {end, actual }}$ & 43.007 & 127.101 & 127.028 \\
\hline$M_{\text {end, regression }}$ & 42.79 & 127.09 & 127.02 \\
\hline$\Delta M_{\text {end, regression }}$ & -0.22 & -0.02 & -0.01 \\
\hline$\Delta_{\text {end, regression }}$ & 0.24 & 0.13 & 0.18 \\
\hline$M_{\text {end, average }}$ & 43.004 & 127.106 & 127.039 \\
\hline$\Delta M_{\text {end, average }}$ & -0.003 & 0.005 & 0.011 \\
\hline$V_{\text {end, average }}$ & 0.014 & 0.011 & 0.013 \\
\hline
\end{tabular}

\footnotetext{
${ }^{a}$ These results were obtained by using the masses listed in Table $3 \Delta$ denotes the difference between the actual end-group mass and the estimated mass. The standard deviations $\sigma_{\mathrm{mon}}$ and $\sigma_{\mathrm{end}}$ were calculated from eqs 6 and $7 . \sigma_{\text {data }}$ is taken to be $60 \mathrm{mu}-(\cdots)-$ denotes the chain of PEG monomer units.
} 
Because the error in the averaging method scales linearly with the error in the experimental data, this method is expected to work for molecular weights up to roughly $\mathrm{m} / \mathrm{z} 10,000$ provided that the measured spectra will be unit mass resolved. The spectra shown clearly indicate that quadrupolar excitation combined with collisional cooling of the cyclotron motion of the ions [32] will be necessary to meet this condition, especially in the higher mass ranges. These techniques will be the subject of future experiments.

\section{End-Group Analysis of a Polymer with Unknown End Group}

The experiments described in the preceding text prove the validity of the end-group determination via MALDI-FTICR-MS. In a second series of experiments, a polymer sample with an unknown end-group structure was investigated. The spectrum of this compound, polyvinyl pyrrolidone (PVP.3000), in Figure 4 shows abundant $[\mathrm{M}+\mathrm{Na}]^{+}$cations. The identity of these sodium adducts was confirmed by comparison of the spectrum with the result of a second measurement in which potassium iodide was added to the matrix (data not shown). The molecular weight distribution again was measured in broadband mode. The masses of the component $[\mathrm{M}+\mathrm{Na}]^{+}$ions (listed in Table 5) were correlated with the degree of polymerization (Figure 5). The resulting end group and monomer masses are given in Table 5. According to the average method the possible end-group (without cation adduct) masses are $60.0568+i^{*} 111.0684$. For $i=0$, the end group mass is indicative of the elemental composition $\mathrm{C}_{3} \mathrm{H}_{8} \mathrm{O}$. Iso-

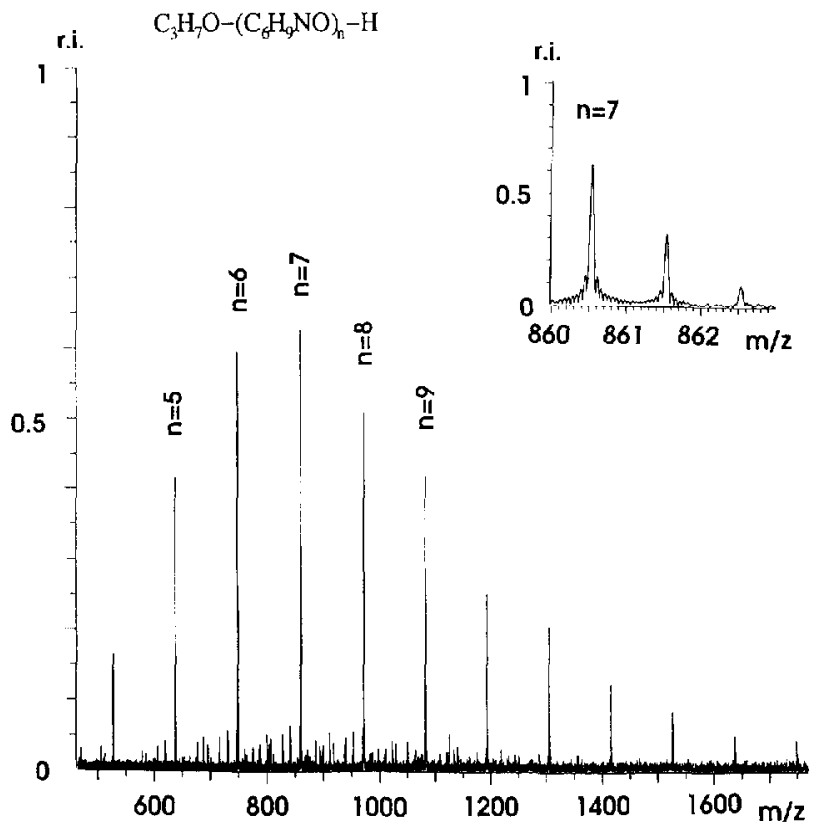

Figure 4. Broadband MALDI-FTICR-MS spectrum of PVP3000 with unknown end-group structure.
Table 5. The measured masses of the components of the PVP3000 molecular weight distributions and the corresponding degree of polymerization $n$, and the masses and deviations obtained from the analysis of these data ${ }^{a}$

\begin{tabular}{lc}
\hline$n$ & {$\left[\left(\mathrm{CH}_{3}\right)_{2} \mathrm{CHO}-(\cdots)-\mathrm{H}+\mathrm{Na}^{+}\right.$} \\
\hline \hline 4 & 527.3247 \\
5 & 638.3927 \\
6 & 749.4606 \\
7 & 860.5268 \\
8 & 971.5933 \\
9 & 1082.6618 \\
10 & 1193.724 \\
11 & 1304.7931
\end{tabular}

Masses and Deviations ( $\mathrm{u}$ )

$M_{\text {mon, actual }}$

111.0684

$M_{\text {mon, regression }}$

111.0667

$\Delta M_{\text {mon, regression }}$

$-0.0017$

$\sigma_{\text {mon, regression }}$

0.0012

$M_{\text {end, actual }}$

83.04728

$M_{\text {end, regression }}$

83.05914

$\Delta M_{\text {end, regressian }}$

0.0118

$\sigma_{\text {end, regression }}$

0.0097

$M_{\text {end, average }}$

83.0465

$\Delta M_{\text {end, average }}$

$-0.0007$

$\sigma_{\text {end, average }}$

0.0028

a $-(\cdots)-$ denotes the chain of PVP monomer units. $\Delta$ denotes the difference between the actual end-group mass and the estimated mass. The standard deviations $\sigma_{\text {mon }}$ and $\sigma_{\text {end }}$ were calculated from eqs 6 and 7.

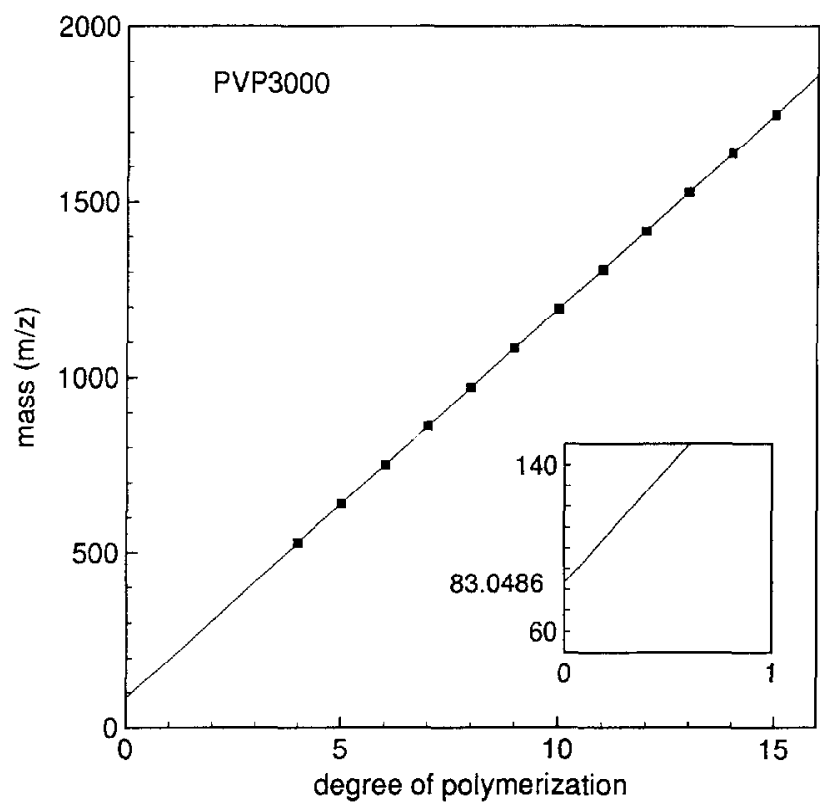

Figure 5. Linear regression on the masses of the PVP3000 component quasimolecular ions versus the corresponding degree of polymerization. 
propanolate anions are well recognized initiators in base-induced polymerization of PVP. In this anionic polymerization reaction the alkyl group of the initiator is incorporated in one of the end groups of the reaction product. Generally these polymerization reactions are quenched by the addition of $\mathrm{H}^{+}$. Therefore we conclude that the end groups of the PVP polymer are $\left(\mathrm{CH}_{3}\right)_{2} \mathrm{CHO}-$ and $-\mathrm{H}$. Given this end-group elemental composition the error in the determined endgroup mass is $12 \mathrm{mu}$. For $i>0$, there are no plausible candidates for the end group of the polymer based on polymer chemical synthesis. Our end-group assignment was confirmed by the supplier.

\section{Acknowledgments}

The authors gratefully acknowledge A. Vijftigschild, M. de Wilde, and I. Stavenuiter for their technical assistance during the various stages of the experiment. We thank Dr. P. Arisz for the derivatization of the native model compounds. This work is part of the research program of FOM and is financially supported by the IAS Instrument Development Program for Advanced Mass Spectrometry and the Foundation for Fundamenteel Onderzoek der Materie (FOM), a subsidiary of the Nederlandse Organisatie voor Wetenschappelijk Onderzoek (NWO, Dutch organization for scientific research). G.J.vR. thanks Bruker-Spectrospin for partial support of a Ph.D. position.

\section{References}

1. Cooper, A. R. Encyclopedia of Polymer Science and Engineering, 2nd ed., Vol. 10, pp 1-19; J. Wiley and Sons, New York, 1985.

2. Johnson, J. F. Encyclopedia of Polymer Science and Engineering, 2nd ed., Vol. 3, pp 491-531; J. Wiley and Sons, New York, 1985.

3. Bletsos, I. V.; Hercules, D. M. Anal. Chem. 1991, 63, 1953.

4. Chait, B. T.; Shpungin, J.; Field, F. H. Int. J. Mass Spectrom. Ion Processes 1985, 58, 121.

5. Johlman, C. L.; Wilkins, C. L.; Hogan, J. D.; Donavan, T. L.; Laude, D. A., Jr.; Youssefi, M. J. Anal. Chem. 1990, 62, 1167.

6. Hsu, A. T.; Marshall, A. G. Anal. Chem. 1988, 60, 932.

7. Beu, S. C.; Senko, M. W.; Quinn, J. P.; Wampler, F. M., III; McLafferty, F. W. J. Am. Soc. Mass Spectrom. 1993, 4, 557.

8. Hillenkamp, F.; Karas, M.; Beavis R. C.; Chait, B. T. Anal. Chem. 1991, 63, 1193A.
9. Chait B. T.; Kent, S. B. H. Science 1992, 257, 1885.

10. Bahr, U.; Deppe, A.; Karas, M.; Hillenkamp, F. Anal. Chem. 1992, 64, 2866.

11. Marshall, A. G.; Comisarow, M. B. J. Chem. Phys. 1976, 64, 110.

12. Marshall, A. G.; Schweikhard, L. Int. J. Mass Spectrom. Ion Processes 1992, 118/119, 37.

13. Sack, R. M.; Gross, M. L. Anal. Chem. 1983, 55, 2419.

14. Sack, R. M.; McGrery, D. A.; Gross, M. L. Anal. Chem. 1985, $57,1290$.

15. Johlman, C. L.; Laude, D. A., Jr.; Wilkins, C. L. Anal. Chem. 1985, 57, 1040.

16. Johlman, C. L.; Laude, D. A., Jr.; Brown, R. S.; Wilkins, C. L. Anal. Chem. 1985, 57, 2726.

17. Holliman, C. L.; Rempel, D. L.; Gross, M. L. Mass Spectrom. Rev. 1994, 13, 105.

18. Koster, C.; Castoro, J. A.; Wilkins, J. A. Anal. Chem. 1993, 65, 2621-2627.

19. Guan, S.; Wahl, M. C.; Wood, T. D.; Marshall, A. G. Anal. Chem. 1993, 65, 1753.

20. Pastor, S. J.; Castoro, J.; Wilkins, C. I. Anal. Chem. 1995, 67, 379.

21. Mclver, R. T., Jr.; Li, Y.; Hunter, R. L. Int. J. Mass Spectrom. Ion Processes 1994, 132, L1.

22. McIver, R. T., Jr.; Li, Y.; Hunter, R. L. Rapid Commun. Mass Spectrum. 1994, 8, 237.

23. Li, Y.; McIver, R. T., Jr. Rapid Commun. Mass Spectrom. 1994, $8,743$.

24. McIver, R. T., Jr.; Li, Y.; Hunter, R. L. Proc. Natl. Acad. Sci. USA, 1994, 91, 4801.

25. Li, Y.; McIver, R. T., Jr.; Hunter, R. L. Anal. Chem. 1994, 66, 2077.

26. de Koster, C. G.; Duursma, M. C.; van Rooij, G. J.; Heeren, R. M. A.; Boon, J. I. Rapid Commun. Mass Spectrom. 1995, 9, 957.

27. Heeren, R. M. A.; Boon, J. J.; Caravatti, P. Proceedings of the 42nd ASMS Conference on Mass Spectrometry and Allied Topics; Chicago, 1994; p 231.

28. Caravatti, P.; Alleman, M. Org. Mass. Spectrom. 1991, $26,514$.

29. Wapstra, A. H.; Audi, G. Nucl. Phys. A 1985, 432, 1.

30. Bevington, P. R. Data Reduction and Error Analysis for the Physical Sciences; McGraw-Hill: New York, 1969.

31. Florry, P. J. J. Am. Chem. Soc, 1940, 62, 1561.

32. Schweikhard, L.; Guan, S.; Marshall, A. G. Int. J. Mass Spectrom. Ion Processes 1992, 120, 71. 